\title{
Detection of glucosamine as a marker for Aspergillus niger: a potential screening method for fungal infections
}

\author{
Christopher L. Allison ${ }^{1} \cdot$ Alex Moskaluk ${ }^{2} \cdot$ Sue VandeWoude ${ }^{2} \cdot$ Melissa M. Reynolds $^{1,3,4}$
}

Received: 7 December 2020 / Revised: 21 January 2021 / Accepted: 8 February 2021 / Published online: 22 February 2021

(C) The Author(s) 2021

\begin{abstract}
Several species of fungus from the genus Aspergillus are implicated in pulmonary infections in immunocompromised patients. Broad screening methods for fungal infections are desirable, as cultures require a considerable amount of time to provide results. Herein, we developed degradation and detection methods to produce and detect D-glucosamine (GlcN) from Aspergillus niger, a species of filamentous fungus. Ultimately, these techniques hold the potential to contribute to the diagnosis of pulmonary fungal infections in immunocompromised patients. In the following studies, we produced $\mathrm{GlcN}$ from fungal-derived chitin to serve as a marker for Aspergillus niger. To accomplish this, A. niger cells were lysed and subjected to a hydrochloric acid degradation protocol. Products were isolated, reconstituted in aqueous solutions, and analyzed using hydrophilic interaction liquid chromatography (HILIC) in tandem with electrospray ionization time-of-flight mass spectrometry. Our results indicated that GlcN was produced from A. niger. To validate these results, products obtained via fungal degradation were compared to products obtained from the degradation of two chitin polymers. The observed retention times and mass spectral extractions provided a two-step validation confirming that $\mathrm{GlcN}$ was produced from fungal-derived chitin. Our studies qualitatively illustrate that GlcN can be produced from A. niger; applying these methods to a more diverse range of fungi offers the potential to render a broad screening method for fungal detection pertinent to diagnosis of fungal infections.
\end{abstract}

Keywords Aspergillus $\cdot$ LC-MS · Chitin · Glucosamine

\section{Introduction}

Aspergillus niger is a ubiquitous species of filamentous fungi $[1,2]$. Colony formation can be visualized after a few days of growth by characteristic black coloration, which is produced by conidial spores [3]. A. niger is a species of filamentous fungus which produces branching hyphae [4] and is widely studied for its ability to produce citric acid, along with

Melissa M. Reynolds

Melissa.reynolds@colostate.edu

1 Department of Chemistry, Colorado State University, 1801 Campus Delivery, Fort Collins, CO 80523, USA

2 Department of Microbiology, Immunology, and Pathology, Colorado State University, 1601 Campus Delivery, Fort Collins, CO 80523, USA

3 Department of Chemical and Biological Engineering, Colorado State University, 1370 Campus Delivery, Fort Collins, CO 80523, USA

4 School of Biomedical Engineering, Colorado State University, 1376 Campus Delivery, Fort Collins, CO 80523, USA industrially significant enzymes such as glucoamylase [5-7]. In addition, it is a well-known plant pathogen [8], and is responsible for black mold formation which can lead to food spoilage in human food sources such as onions and grapes [5]. Occasionally, A. niger can be an opportunistic human pathogen [9]. Inhalation of spores can lead to pulmonary aspergillosis, which usually occurs in immunocompromised patients [10-12]. Diagnosis of respiratory aspergillosis remains problematic, leading to delays in treatment and high mortality rates [13]. One of the primary structural constituents of filamentous fungi is chitin, a $\beta-1-4$-linked homopolymer of GlcNAc residues [14]. In the following studies, D-glucosamine (GlcN) produced from structural chitin in A. niger and used as a molecular indicator for the presence of fungus. Ultimately, these methods hold the potential to screen for the presence of filamentous fungi, and play a role in fungal infection diagnostics.

Chitin is a $\beta$-1-4-linked homopolymer of GlcNAc residues [14]. It is the second most abundant natural polysaccharide in the world, behind cellulose $[15,16]$. Chitin is a component of crustacean and arthropod exoskeletons and is found in fungal cell walls, where it confers rigidity to its parent organism [17]. 
It exists in nature as nearly straight microfibrils with average diameters of $\sim 2.8 \mathrm{~nm}$ and of indeterminate lengths [18]. Its size has been reported from $\sim 100$ GlcNAc residues in yeast to 5-8000 residues in crab exoskeletons [19]. While most species of yeasts contain only $1-2 \%$ chitin, its abundance in filamentous fungi ranges from 10 to $30 \%$ [14]. Chitin is found in the cell walls and septa of all species of pathogenic fungi [20]. Some fungi produce chitin deacetylase enzymes that modify chitin to chitosan during biosynthesis; however, chitosan's abundance in most fungi has not been welldefined [14, 21, 22].

Chitin is ubiquitous in the cell walls of fungi but is not found endogenously in humans. While GlcN can be found endogenously, the introduction of an aqueous extraction prior to the production of chitin-derived GlcN would effectively remove residual species, as chitin is insoluble prior to modification. In the following experiments, we tested fungalderived chitin degraded from Aspergillus niger, a filamentous fungal species closely related to A. fumigatus, and characterized its degradation products using LC-MS. Upon exposure to $\mathrm{HCl}$, chitin is simultaneously depolymerized and deacetylated as shown in Fig. 1, resulting in GlcN monomers and oligomers that can be related back to the presence of the parent polymer. These residues are soluble in aqueous liquids and can be separated and detected using LC-MS. Our research indicates that chitin-derived GlcN can be produced from $A$. niger, a species that has been implicated in fungal pulmonary infections. To accomplish this, we obtained $A$. niger cells and developed sample preparation steps to separate extracellular and intracellular components. We subjected fungi to cell lysis and degradation protocols, then used LC-MS to characterize the degradation products. We found that GlcN was quickly produced from chitin via simultaneous depolymerization and deacetylation of these intracellular polysaccharides.

Fewer than $1 \%$ of fungal species are potentially pathogenic [23]. Fungal spores are found ubiquitously in the environment, and most individuals are exposed to large numbers of airborne fungi with no notable health repercussions. However, a minority of fungal species are extremely effective opportunistic pathogens [24]. Worldwide, over 300 million cases of serious fungal infections occur annually [25]. Fungal infections can occur superficially (e.g., athlete's foot) or as systemic infections such as candidemia or aspergillosis [26-29]. Pulmonary fungal infections comprise a significant number of systemics fungal diseases, with global incidence of $>$ $10,000,000$ patients. $1,400,000$ of these result in fatalities annually [25]. A variety of genera such as Aspergillus, Cryptococcus, and Pneumocystis are responsible for these infections [30]. Chronic pulmonary aspergillosis is listed by The Global Action Fund for Fungal Infections (GAFFI) as one of four "priority fungal infections." Disease is most often caused by Aspergillus fumigatus but can also be caused by A. niger or A. flavus [30]. Chronic pulmonary aspergillosis (CPA) is most frequently seen in patients that have at one time had lung disease, such as tuberculosis, chronic obstructive pulmonary disease, or lung cancer [31].

CPA progresses rapidly in immunocompromised patients, resulting in high mortality rates and necessitating prompt treatment $[32,33]$. We propose that liquid chromatographymass spectrometry (LC-MS) can be used as a broad screening method to assist in diagnosing pulmonary fungal infections (e.g., CPA) via the detection of chitin contained within fungal cell walls. Sputum or lavage samples from patients with pulmonary fungal infections contain fungi [34, 35], facilitating noninvasive sample acquisition. Chitin may be detected via the separation and detection of GlcN monomers and oligomers that are produced during the controlled degradation of chitin. Given this, detecting polymeric chitin has the potential to serve as a screening method for pulmonary fungal infections in clinical settings. Our results are significant as they corroborate methods of chemical modification and instrumental detection which can be used in tandem to detect fungalderived GlcN. Our instrument configuration was chosen to provide a straightforward qualitative analysis that fungalderived GlcN can be produced from the chemical modification of $A$. niger and can subsequently be detected using LC-
Fig. 1 Simultaneous chemical modifications that occur upon the exposure of chitin to $\mathrm{HCl}$. Polymers are simultaneously depolymerized and deacetylated, producing a low molecular weight chemical fingerprint that consists primarily of GlcN
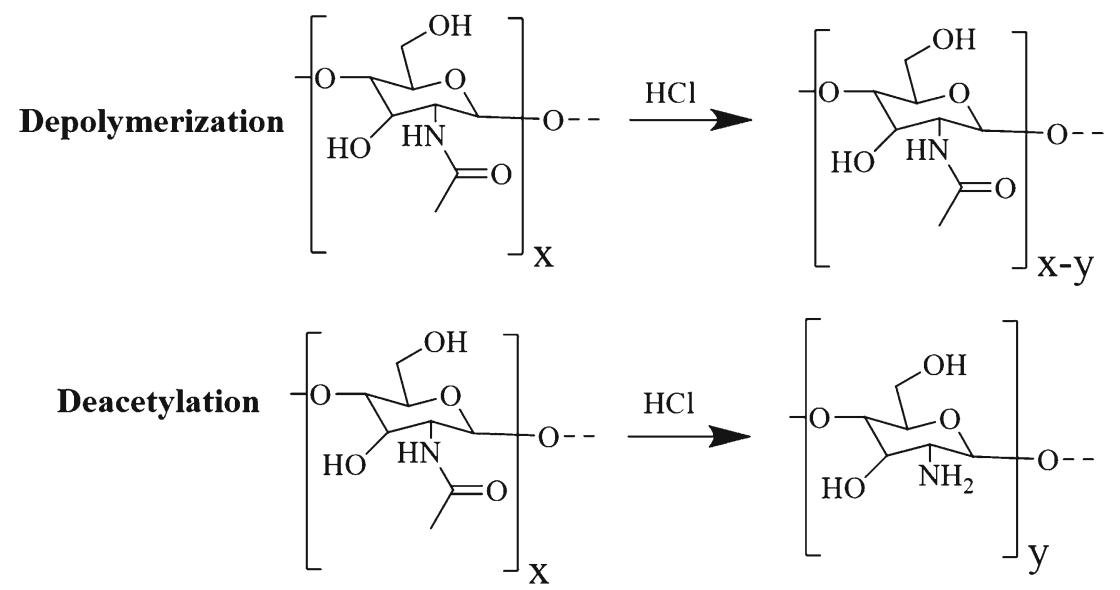
MS. Having established the efficacy of our methods, we suggest that future experiments approaching clinical applicability test our methods using an LC-MS configuration common in clinical laboratories, such as an LC-MS/MS. Eventually, we hope that these methods will be implemented clinically as part of fungal infection diagnostics.

\section{Materials and methods}

\section{Materials}

A. niger fungi were obtained from domestic cat hair samples and cultured on an agar medium. D-Glucosamine hydrochloride was obtained from MP Biomedicals (Santa Ana, CA). Chitin polymer (100\% acetylated) and $N$-acetyl-D-glucosamine (> 98.0\%) were obtained from TCI (Portland, OR). Chitin polymer (20-30\% deacetylated) was obtained from Alfa Aesar. ACS-grade acetone and Optima LC-MS grade formic acid were obtained from Fisher Chemical (Waltham, MA). Hydrochloric acid was obtained from Ward's Science (Rochester, NY). LC-MS grade water, LC-MS grade acetonitrile, low molecular weight chitosan (96\% deacetylated), and ammonium acetate (>98.0\%) were obtained were obtained from EMD Millipore (Burlington, MA). 0.2- $\mu \mathrm{m}$ Captiva Econofilters were obtained from Agilent (Palo Alto, CA). C18 Macro spin columns were obtained from Harvard Apparatus (Holliston, MA).

\section{Sample preparation}

A. niger was grown and maintained on Sabouraud dextrose agar at $22{ }^{\circ} \mathrm{C}$ until harvesting. The fungal culture was originally obtained from domestic cat hair sample and cultured on a dermatophyte test media (DTM) agar plate via standard toothbrush method. The identification of the fungi was performed using colony morphology and Internal Transcribed Spacer 2 (ITS-2) region sequencing. To obtain ITS-2 sequencing results, fungus was harvested for DNA extraction and used for conventional PCR analysis. PCR products were sent for sequencing at Psomagen and sequences were compared to entries in GenBank. A. niger cells were transferred into $15-\mathrm{mL}$ centrifuge tubes in a biosafety hood. Five milliliters chilled acetone $\left(-20^{\circ} \mathrm{C}\right)$ was added prior to removal from the hood. Samples were incubated for $60 \mathrm{~min}$ at $-20{ }^{\circ} \mathrm{C}$. Following incubation, samples were vortexed for $30 \mathrm{~s}$ then centrifuged for $10 \mathrm{~min}$ at $15 \mathrm{k} \times g$. The supernatant was removed, and samples were allowed to stand for $30 \mathrm{~min}$ to encourage the evaporation of residual acetone. Liquid nitrogen was added directly to the dried pellets to lyse cells. After $5 \mathrm{~min}, 5 \mathrm{~mL}$ of $-20{ }^{\circ} \mathrm{C}$ acetone was added and the pellet vortexed again for $30 \mathrm{~s}$. The supernatant was decanted, and the pellet was allowed to evaporate for $30 \mathrm{~min}$. Four milliliters
Table 1 Polymers used for degradation studies

\begin{tabular}{llll}
\hline Polymer & Supplier & $\%$ GlcN & $\%$ GlcNAc \\
\hline Chitin 1 & Alfa Aesar & $20-30^{\mathrm{a}}$ & $70-80$ \\
Chitin 2 & TCI & - & $100^{\mathrm{b}}$ \\
\hline
\end{tabular}

${ }^{\mathrm{a}}$ Alfa Aesar certificate of analysis; ${ }^{\mathrm{b}} \mathrm{TCI}$ certificate of analysis

$10 \mathrm{M} \mathrm{HCl}$ was added to the dried pellet. The pellet was vortexed, and the resulting suspension was transferred to a round-bottom flask. The flask was heated in a water bath held at $90{ }^{\circ} \mathrm{C}$ and the acid evaporated over $\sim 6 \mathrm{~h}$. Following evaporation, the dried material in the flask was reconstituted using Milli-Q grade water $(18.2 \mathrm{M} \Omega \bullet \mathrm{cm})$. Aliquots were drawn from the flask and passed through $0.2-\mu \mathrm{m}$ filters. The eluent was added to $\mathrm{C} 18$ spin columns and centrifuged for $4 \mathrm{~min}$ at $2 \mathrm{k} \times g$. The filtrate was collected and analyzed via LC-MS.

\section{LC-MS methods}

LC-MS analyses were carried out on an Agilent 6224 time-offlight mass spectrometer coupled to an Agilent 1260 binary liquid chromatograph (Agilent, Palo Alto, CA). Separations were performed using a Thermo Fisher Acclaim HILIC-10 column with dimensions of $4.6 \times 150 \mathrm{~mm}$ and $5-\mu \mathrm{m}$ particle sizes. The mobile phase was composed of LC-MS grade water and acetonitrile (ACN). Each mobile phase component contained $10 \mathrm{mM}$ ammonium acetate and $0.05 \%$ formic acid with a final $\mathrm{pH}$ 4.0. The total length of chromatography runs was $50 \mathrm{~min}$. Solvent flow rate was set to $0.350 \mathrm{~mL} / \mathrm{min}$ for the duration of the separation. A gradient elution was used with initial solvent proportions of 90:10 ACN: $\mathrm{H}_{2} \mathrm{O}$. The solvent ratio was adjusted to $80: 20 \mathrm{ACN}: \mathrm{H}_{2} \mathrm{O}$ from 0 to $30 \mathrm{~min}$. From 30 to $31 \mathrm{~min}$, the solvent ratio was returned to $90: 10$ $\mathrm{ACN}: \mathrm{H}_{2} \mathrm{O}$. From 31 to $50 \mathrm{~min}$, the column was allowed to reequilibrate and the baseline stabilize. The ion source used was a dual electrospray ionization source operating in positive ionization mode. Ion source conditions were as follows: $3500 \mathrm{~V}$ capillary voltage, $120 \mathrm{~V}$ fragmentor voltage, $60 \mathrm{~V}$ skimmer voltage, $250 \mathrm{~V}$ octupole voltage, $10 \mathrm{~L} \mathrm{~min}^{-1}$ gas flow $\left(\mathrm{N}_{2}\right)$ at $300{ }^{\circ} \mathrm{C}$, and $45 \mathrm{psig}$ nebulizer pressure. The detection range was set to $95-3200 \mathrm{~m} / \mathrm{z}$.

Table 2 Ions observed in MS extractions from LC-MS of GlcN standards

\begin{tabular}{ll}
\hline Signal observed $(m / z)$ & Adduct \\
\hline 162 & {$\left[\mathrm{C}_{6} \mathrm{H}_{13} \mathrm{NO}_{5}-\mathrm{H}_{2} \mathrm{O}+\mathrm{H}\right]^{+}$} \\
180 & {$\left[\mathrm{C}_{6} \mathrm{H}_{13} \mathrm{NO}_{5}+\mathrm{H}\right]^{+}$} \\
202 & {$\left[\mathrm{C}_{6} \mathrm{H}_{13} \mathrm{NO}_{5}+\mathrm{Na}\right]^{+}$} \\
381 & {$\left[(2) \mathrm{C}_{6} \mathrm{H}_{13} \mathrm{NO}_{5}+\mathrm{Na}\right]^{+}$} \\
\hline
\end{tabular}




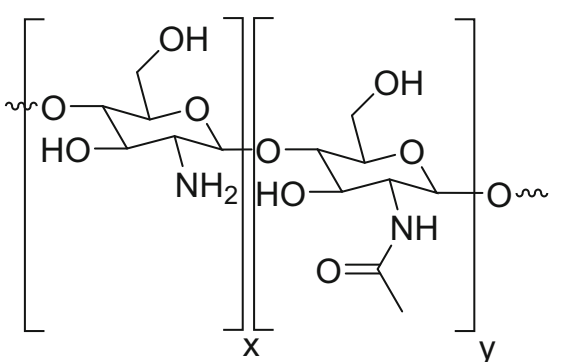

Fig. 2 Generic structure of chitin (>50\% GlcNAc) showing GlcN (x) and GlcNAc (y) subunits

\section{LC-MS of controls and samples}

Chitin polymer 1 and chitin polymer 2 were subjugated to $\mathrm{HCl}$ degradation (see Table 1 for the specific properties of these polymers). For LC-MS analysis of polymer degradation products, $\sim 4 \mathrm{mg}$ chitin polymer 1 and chitin polymer were 2 were added to a 50-mL round-bottomed flask. An appropriate quantity of $10 \mathrm{M} \mathrm{HCl}$ was added to the powdered samples. The flask was heated in a water bath held at $90{ }^{\circ} \mathrm{C}$ and the acid evaporated over $\sim 6 \mathrm{~h}$. Following evaporation, the dried material in the flask was reconstituted using Milli-Q grade water $(18.2 \mathrm{M} \Omega \bullet \mathrm{cm})$. Aliquots were drawn from the flask and passed through $0.2-\mu \mathrm{m}$ filters. The filtrate was collected and analyzed via LC-MS. All experiments were performed in triplicate.

\section{Data analysis}

All data were analyzed using Agilent MassHunter Qualitative Analysis B.07.00 software. ESI optimization was performed prior to these experiments and a library of potential degradation products was generated to enable extracted ion chromatogram (EIC) scans for data deconvolution. Degradation experiments performed on A. niger samples were compared to the LC-MS results for chitin polymer degradations. Retention times and accurate mass measurements were both accounted for to provide a two-step validation confirming the identity of degradation products.

\section{Results and discussion}

\section{Analysis of GIcN standards and chitin polymers}

GlcN standards were purchased from MP Biomedicals. Standards were dissolved in LC-MS grade water to a concentration of $1 \mathrm{mg} / \mathrm{mL}$ and analyzed using LC-MS. Ions that were observed are listed in Table 2. Chromatograms and mass spectra associated with these results can be found in the Supplementary Information (ESM).

While it was expected that the most prevalent ion would be a protonated molecule at $180 \mathrm{~m} / \mathrm{z}$, the most abundant ion in mass spectra was shown at $381 \mathrm{~m} / \mathrm{z}$. To the best of our knowledge, dimerization has not been previously reported in ESI analysis of GlcN; however, $[2 \mathrm{M}+\mathrm{H}]^{+},[2 \mathrm{M}+\mathrm{Na}]^{+}$, and variations of these dimers have been observed in other compounds [36, 37]. Dimerized ESI ions are proposed to result from noncovalent interactions between residues in solution, as Coulombic barriers preclude dimerization following electrospray ionization [38]. One hundred sixty-two, 180, and $202 \mathrm{~m} / \mathrm{z}$ are commonly seen ions in ESI analysis of GlcN. $162 \mathrm{~m} / \mathrm{z}$ occurs following the dehydration and protonation of GlcN and has previously been observed in the ESI analysis of GlcN [39]. $180 \mathrm{~m} / \mathrm{z}$ is a protonated GlcN molecule

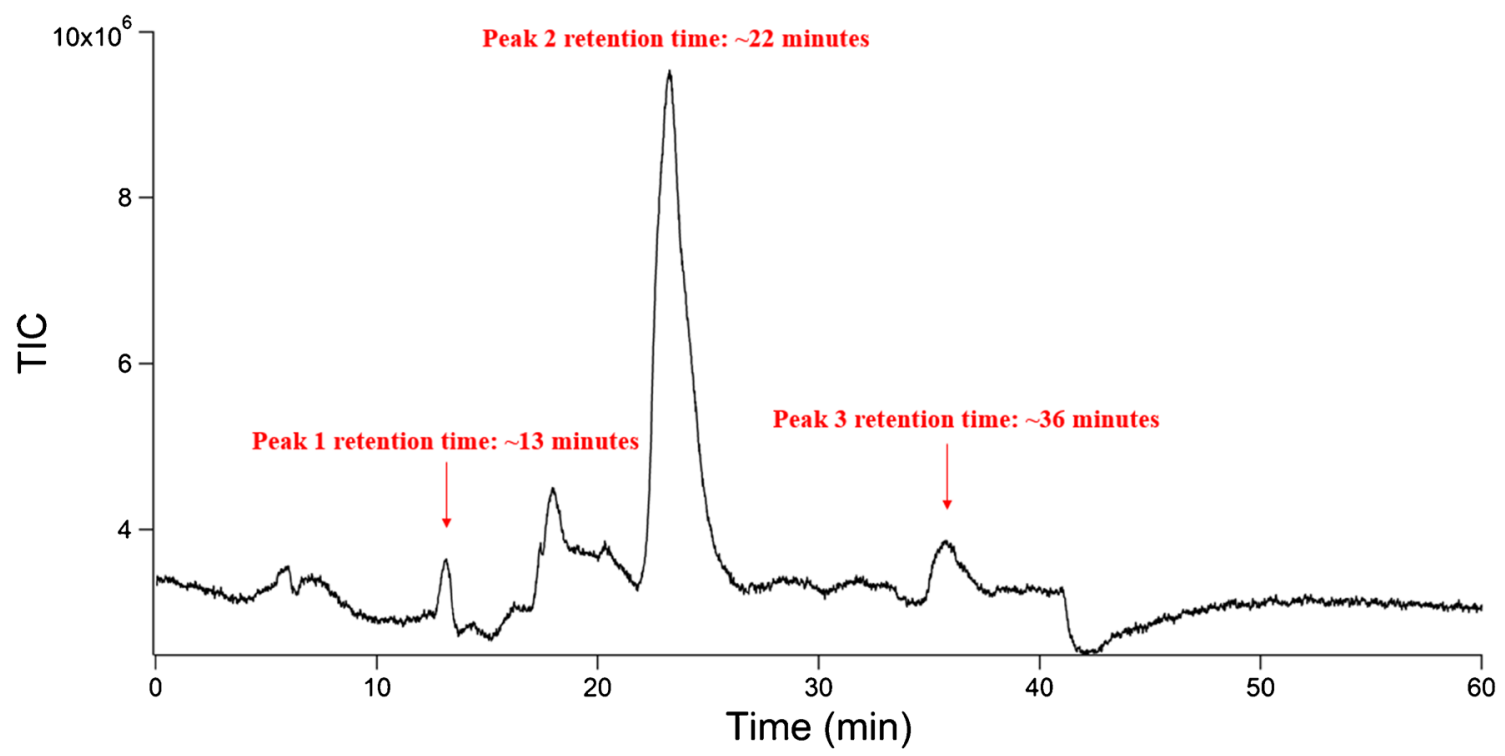

Fig. 3 Representative chromatogram of chitin polymer 1 degradation products. Three peaks contained $\mathrm{m} / \mathrm{z}$ that indicated the presence of the degradation products of chitin. Unlabeled peaks did not contain $\mathrm{m} / \mathrm{z}$, that correlated to chitin degradation products 
Table 3 Summary of peaks and the ions contained within these

\begin{tabular}{lll}
\hline Retention time & Compounds & $m / z$ identified \\
\hline $13 \mathrm{~min}$ & GlcNAc & $204,222,244,465$ \\
$24 \mathrm{~min}$ & GlcN & $162,180,202,381$ \\
$36 \mathrm{~min}$ & GlcN dimers & $341,363,703$ \\
\hline
\end{tabular}

for which formation is promoted by the acidic $\mathrm{pH}$ in the mobile phase used. $202 \mathrm{~m} / \mathrm{z}$ represents a sodiated adduct.

The efficacy of degradation methods was established via identification of the degradation products from chitin polymers following their exposure to $\mathrm{HCl}$ degradation protocols. In these experiments, two chitin polymers were exposed to $\mathrm{HCl}$ degradation protocols and the products were characterized using LC-MS. Information regarding the polymers used for degradation studies can be found in Table 1, along with a generic structure of chitin/chitosan shown in Fig. 2.

The first polymer used in these studies had a composition of 75:25 GlcNAc to GlcN residues. This polymer was exposed to $5 \mathrm{M} \mathrm{HCl}$ at a concentration of $1 \mathrm{mg} / \mathrm{mL}$ in a roundbottom flask. The acid was removed under heat and evaporation over approximately $6 \mathrm{~h}$. Milli-Q grade water $(18.2 \mathrm{M} \Omega \bullet \mathrm{cm})$ was added to the dry flask to resuspend soluble species. The resulting suspension was removed, filtered, and analyzed via LC-MS. The chromatogram obtained from these experiments is shown in Fig. 3.

Three peaks in the chromatograms obtained from these experiments contained $\mathrm{m} / \mathrm{z}$ indicating the presence of the degradation products of chitin. The first of these peaks eluted at 13 min and contained GlcNAc. The most prominent peak in this chromatogram eluted at $\sim 24$ min and contained GlcN. A third peak eluting at $\sim 36$ min contained GlcN dimer adducts.
The peak containing GlcNAc was small in comparison to the GlcN peak, which was expected given the ability of $\mathrm{HCl}$ to deacetylate GlcNAc residues. Concentrated $\mathrm{HCl}$ depolymerizes chitin polymers considerably faster than it deacetylates them [39]. Hence, observation of acetylated species was not surprising. GlcN was predicted to be the prevalent product from this degradation protocol, given it is the final product of the depolymerization and deacetylation of chitin polymer. This prediction was confirmed by the relative size of the peak eluting at $24 \mathrm{~min}$. Given the propensity of concentrated acid to deacetylate chitin more rapidly than it deacetylates GlcNAc residues, the size of this peak also indicated that depolymerization was largely complete by the time the $\mathrm{HCl}$ had evaporated. The peak at 36 min containing GlcN-dimerized adducts was relatively small in size and was representative of fully deacetylated, partially depolymerized residues. A summary of the peaks and the compounds eluting in these can be seen in Table 3. Mass spectral assignments for each peak can be found in the ESM.

The second polymer used exposed to the $\mathrm{HCl}$ degradation protocol was chitin polymer 2 , which had a composition of $100 \%$ GlcNAc. These studies mirrored those with chitin polymer 1 , with this analyte being exposed to $10 \mathrm{M} \mathrm{HCl}$ at a concentration of $1 \mathrm{mg} / \mathrm{mL}$ in a round-bottomed flask. The acid was removed under heat by evaporation over approximately $6 \mathrm{~h}$. Milli-Q grade water $(18.2 \mathrm{M} \Omega \bullet \mathrm{cm})$ was added to the dry flask to resuspend soluble species. The resulting suspension was removed, filtered, and analyzed via LC-MS. The chromatogram obtained from these experiments is shown in Fig. 4.

Two peaks in the chromatograms obtained from these experiments contained $\mathrm{m} / \mathrm{z}$ representative of chitin's degradation products. The first of these eluted at $13 \mathrm{~min}$ and contained GlcNAc. The second peak eluted at $24 \mathrm{~min}$ and contained

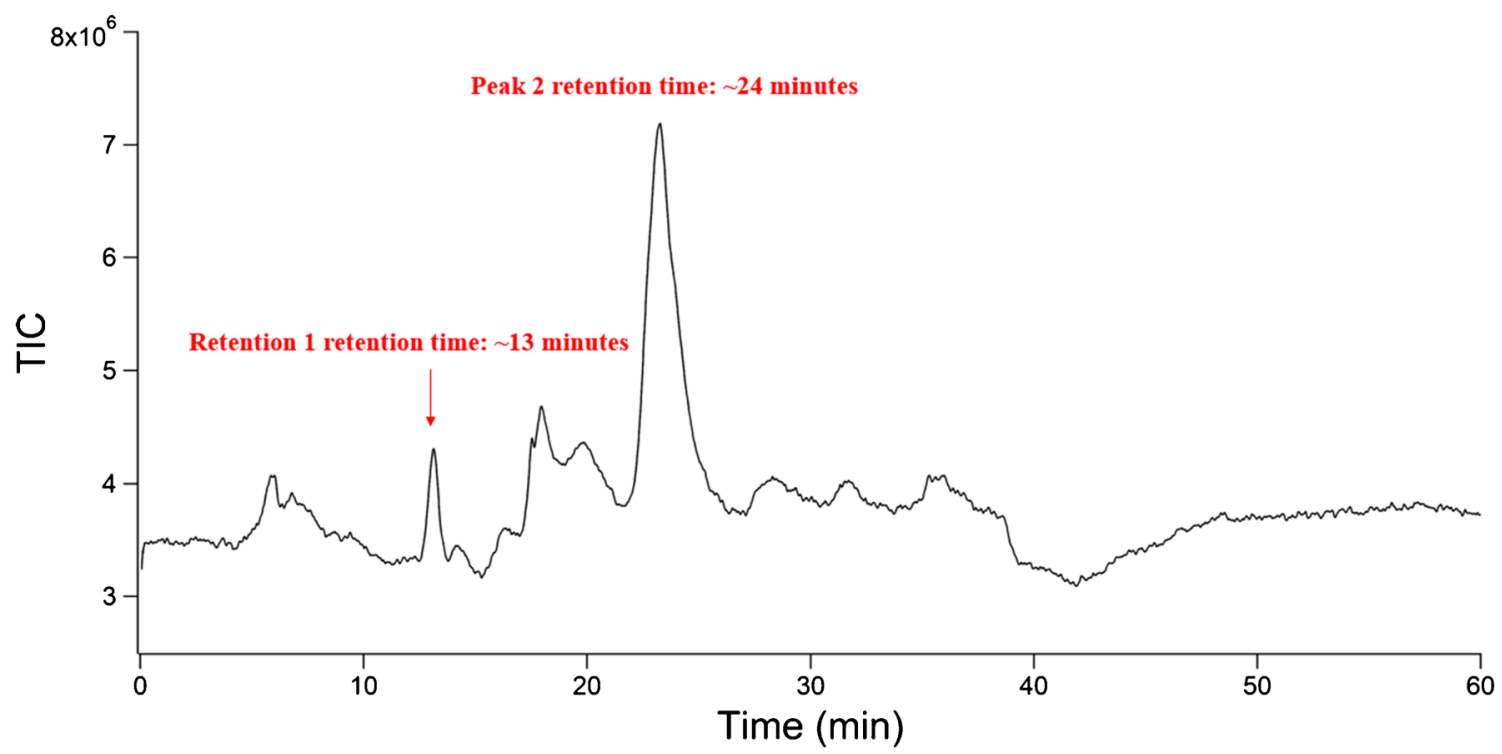

Fig. 4 Chromatogram of chitin polymer 2 degradation products. Two peaks contained $\mathrm{m} / \mathrm{z}$, that indicated the presence of chitin degradation products. Remaining peaks did not contain $\mathrm{m} / \mathrm{z}$, representative of chitin degradation products 
Table 4 Summary of peaks and the ions contained within these

\begin{tabular}{lll}
\hline Retention time & Compounds & $m / z$ identified \\
\hline 13 min & GlcNAc & $204,222,244,465$ \\
24 min & GlcN & $162,180,202,381$ \\
\hline
\end{tabular}

GlcN. The peak at 13 min (containing GlcNAc) was small in comparison to the peak at $24 \mathrm{~min}$ (containing GlcN). Given the ability of $\mathrm{HCl}$ to deacetylate $\mathrm{GlcNAc}$, we expected a lower quantity of GlcNAc to be present. These results were consistent with those from chitin polymer 1 . A third peak at $36 \mathrm{~min}$ contained GlcN dimers; however, these results have not been included as the peak barely protruded above the baseline. A summary of the peaks and the compounds eluting in these can be seen in Table 4. Mass spectral assignments for both peaks can be found in the ESM.

\section{Analysis of degradation products from $A$. niger}

Following the testing of LC-MS methods using chitin polymers, the ability of degradation methods in tandem with LCMS to detect fungal-derived GlcN was explored. A. niger cells were used for these studies. Cell lysis and precipitation steps were performed first, followed by solid-phase extraction steps for sample cleanup. Following this, we analyzed the degradation products using LC-MS. A representative chromatogram from these experiments can be seen in Fig. 5.

The unedited chromatogram obtained from the extraction and degradation of chitin from $A$. niger contained multiple peaks that overlapped in areas from 5 to $25 \mathrm{~min}$. As a HILIC HPLC column was used, it was inferred that compounds eluted from least hydrophilic to most hydrophilic. The final peak eluted at $\sim 24 \mathrm{~min}$ and contained $\mathrm{GlcN}$, along with $\mathrm{m} / \mathrm{z}$ representing coeluting compounds. An extracted ion chromatogram (EIC) for the accurate mass $\mathrm{m} / \mathrm{z}$ measurement of a protonated $\mathrm{GlcN}$ molecule was performed, and the peak was overlaid with those from chitin polymer degradation experiments. EICs were performed in this instance to specifically highlight the predicted degradation product from the degradation of chitin, and to validate that polymer models and fungi both produce GlcN during degradation. The EIC overlays confirm the production of GlcN from both chitin polymers studied, as well as the chitin contained in A. niger cells. The chromatographic peaks for both chitin polymers as well as fungal extractions line up at $\sim 24 \mathrm{~min}$. This overlay can be seen in Fig. 6. Mass spectra were extracted from these peaks, which can be found in Fig. 7. Chromatographic elution times and analysis of $\mathrm{m} / \mathrm{z}$ values suggest the presence of ions with identical polarity and molecular weights, providing a two-step validation of the presence of GlcN in all samples tested.

Extractions of mass spectra from chromatographic peaks at $\sim 24 \mathrm{~min}$ all displayed $\mathrm{m} / \mathrm{z}$ at 180 , indicative of ions with formulas matching those of protonated GlcN molecules, $\left[\mathrm{C}_{6} \mathrm{H}_{13} \mathrm{NO}_{5}+\mathrm{H}\right]^{+}$. The degradation of both chitin polymers displayed a $\mathrm{m} / \mathrm{z}$ at 202 , indicative of ions with formulas matching those of sodiated GlcN adducts, $\left[\mathrm{C}_{6} \mathrm{H}_{13} \mathrm{NO}_{5}+\right.$ $\mathrm{Na}]^{+}$. The mass spectrum of $A$. niger contained a $\mathrm{m} / \mathrm{z}$ at 202; however, its abundance was very low. The degradation products from chitin polymers also displayed a $\mathrm{m} / \mathrm{z}$ at 162 , indicative of ions with formulas matching those of protonated dehydrated $\mathrm{GlcN},\left[\mathrm{C}_{6} \mathrm{H}_{13} \mathrm{NO}_{5}-\mathrm{H}_{2} \mathrm{O}+\mathrm{H}\right]^{+}$. While the mass spectrum of $A$. niger contained a $\mathrm{m} / \mathrm{z}$ at 162 , its monoisotopic mass varied from that expected from a protonated dehydrated

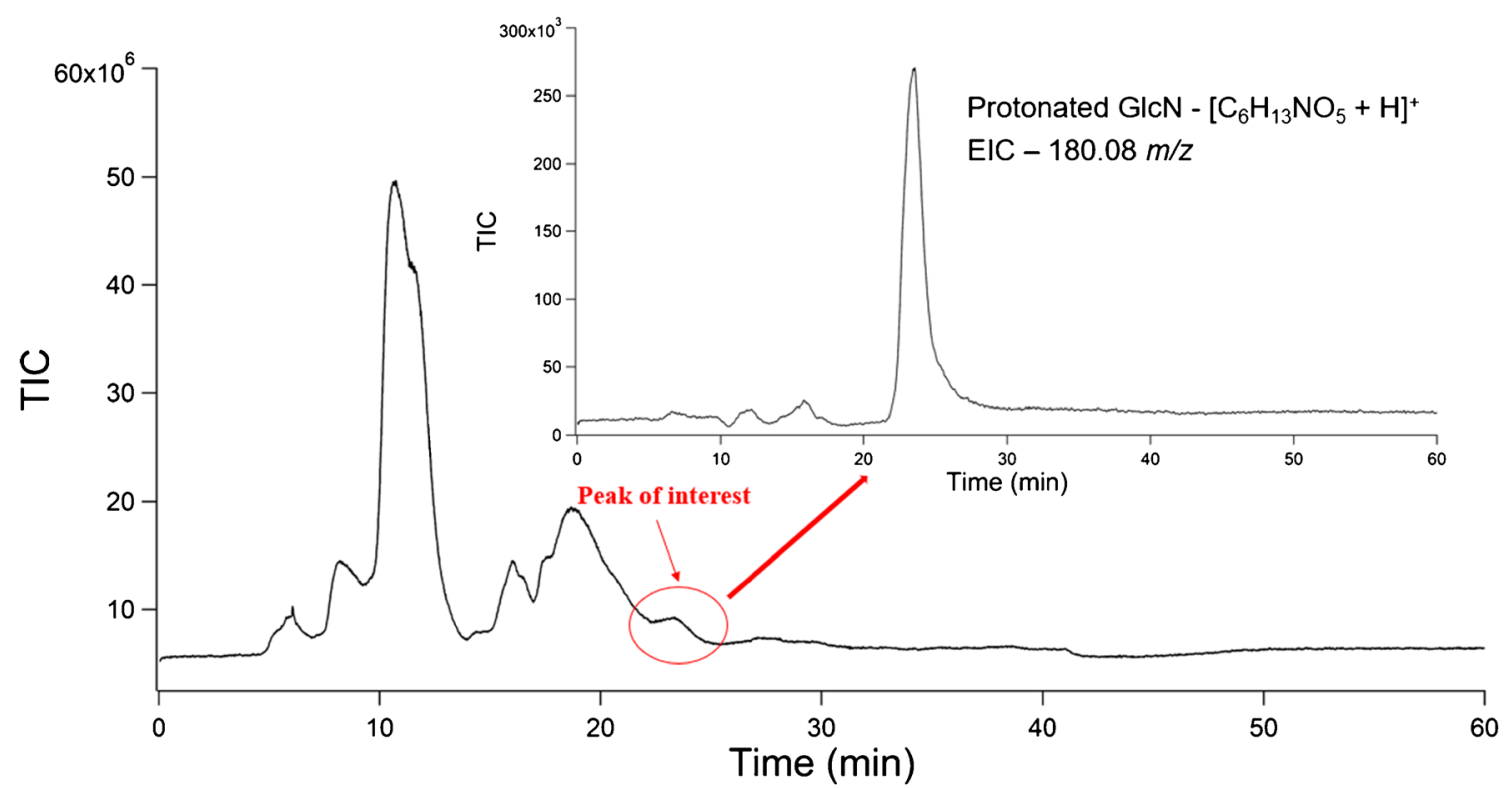

Fig. 5 Chromatogram of A. niger degradation products. Analytes of interest eluted in a broad peak at $\sim 24$ min. The inset shows an extracted ion chromatogram for the monoisotopic mass of GlcN, 180.08 


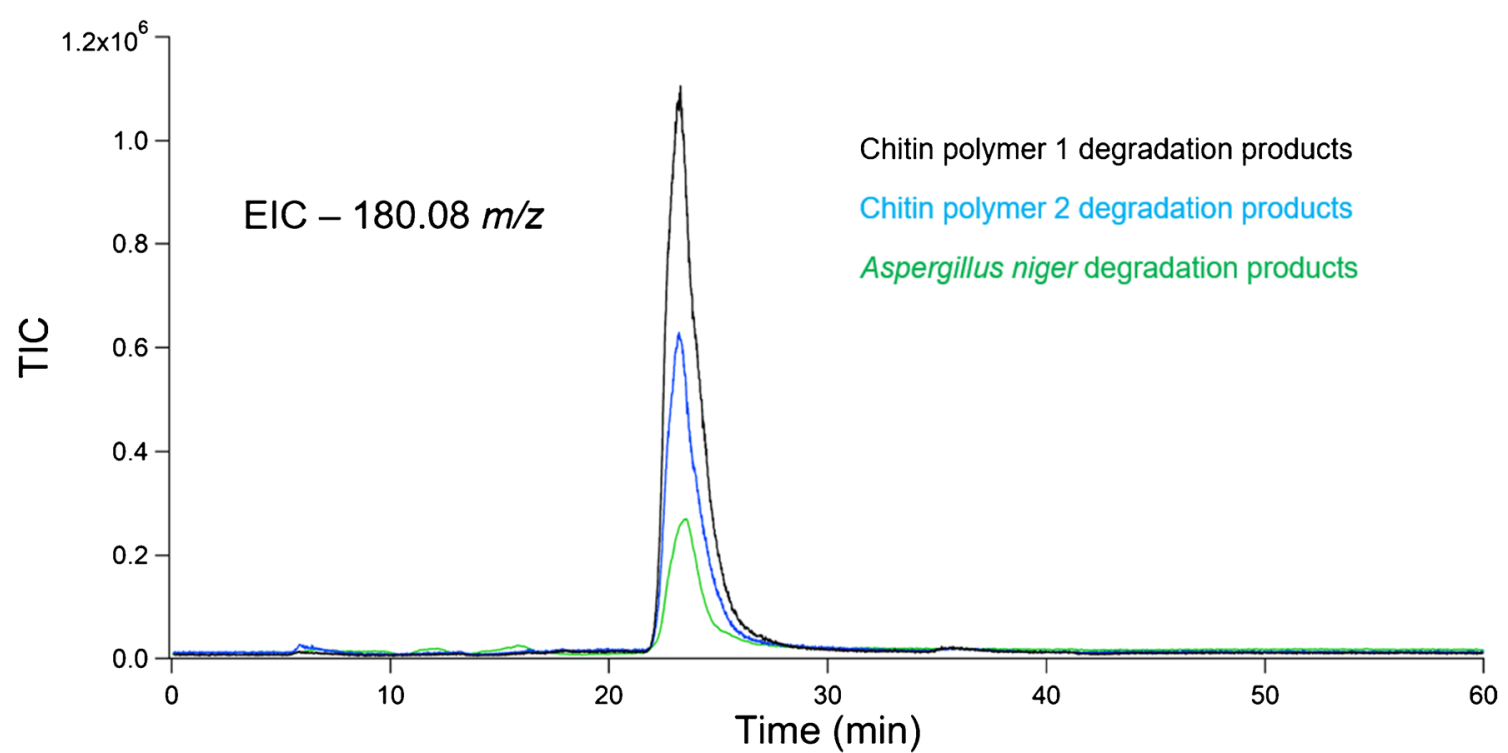

Fig. 6 Overlay of EICs from degradation experiments. The value for EIC scans was set to 180.08 to highlight the elution of protonated GlcN. The black trace represents GlcN produced from chitin polymer 1 . The blue

$\mathrm{GlcN}$ ion. Closer examination revealed that the peak at $162.1109 \mathrm{~m} / \mathrm{z}$ was split, suggesting that two molecules with similar but not identical $\mathrm{m} / \mathrm{z}$ ratios were present in mass spectra. Greater resolving power would be required necessary to distinguish between these peaks.

Further studies to differentiate between endogenous and exogenous GlcN derived from chitin will be necessary prior to implementation of these methods in clinical facilities. Separating GlcN from these sources could be done using several approaches: by aqueous liquid extraction, by sub-micron filtration, or by dialysis. In addition, centrifugation has been shown to be an effective means of concentrating fungal spores trace represents GlcN produced from chitin polymer 2. The green trace represents GlcN produced from A. niger fungi

[40]. Regardless of the approach chosen, a crucial aspect of separating endogenous and exogenous GlcN is that endogenous $\mathrm{GlcN}$ is removed prior to the production of $\mathrm{GlcN}$ from chitin, thereby reducing the likelihood of false positives. Use of an aqueous extraction step prior to the degradation of chitin would remove endogenous GlcN, given its high solubility. Alternatively, isolating fungi immediately following sample acquisition could be accomplished by sub-micron filtration, dialysis, or centrifugation and recovery of spores and other insoluble species prior to their exposure to $\mathrm{HCl}$. The detection of GlcN by LC-MS/MS systems has been performed in multiple studies, with limits of quantitation at or below $10 \mathrm{ng} / \mathrm{mL}$

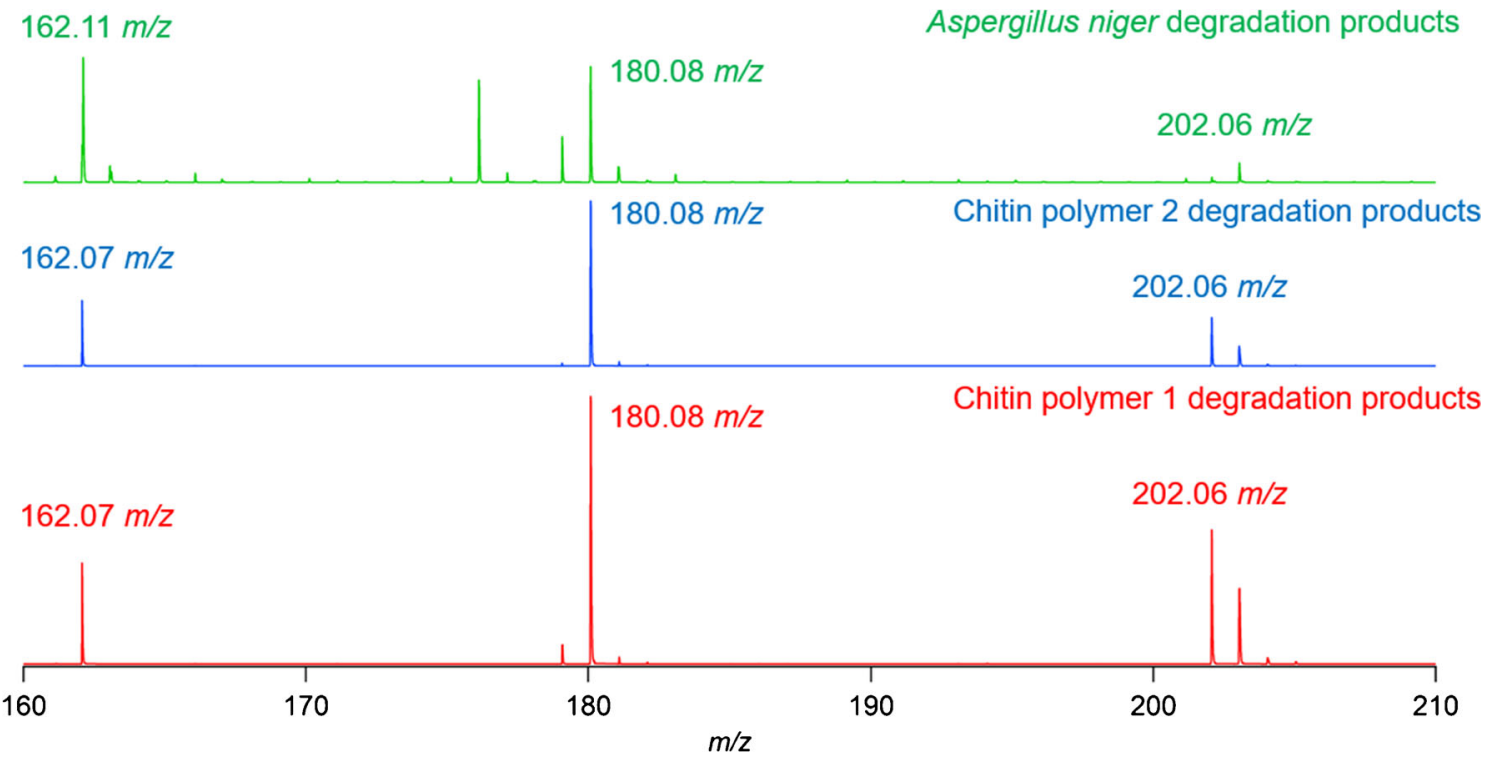

Fig. 7 MS overlays from LC-MS analysis of polymer and fungal degradations. MS extractions from chitin polymer 1 are shown in black. MS extractions from chitin polymer 2 are shown in blue. MS extractions from A. niger are shown in green 
[41-43]. For our qualitative application, these values indicate limits of detection for GlcN in biologically relevant matrices as low as $\sim 3 \mathrm{ng} / \mathrm{mL}$ for clinically relevant LC-MS systems. Ultimately, we hope that the methods presented herein may serve as a key component in the rapid detection of fungi.

\section{Conclusions}

In our studies, we used LC-MS to detect GlcN from fungalderived chitin. To accomplish this, A. niger fungi were obtained and exposed these to cell lysis and sample cleanup steps. Lysed cells were exposed to previously developed degradation protocols using $\mathrm{HCl}$. The acidic solution was evaporated, and water-soluble analytes were resuspended into an aqueous suspension. These were filtered and analyzed using HILICESI-MS. LC-MS analysis indicated the presence of a chromatographic peak with a retention time matching that of GlcN produced from the degradation of chitin. Mass spectral extractions of this peak provided secondary confirmation of its identity, showing a $\mathrm{m} / \mathrm{z}$ at 180.08 , matching that of a protonated GlcN molecule. Chitin degradation methods were developed to degrade fungal-derived chitin and to produce GlcN. The analyte produced from fungal degradations matched those generated during the analysis and comparison of degradation protocols using several variations of chitin and chitosan. Retention times and accurate mass measurements were compared to validate that chitosan polymers, chitin polymers, as well as A. niger produce $\mathrm{GlcN}$ following exposure to $\mathrm{HCl}$. Cumulatively, the novelty of our results lies in our combination of chemical modification methods and analytical detection methods (HILIC-ESI-MS) to produce and detect GlcN from A. niger. By applying reproducible methods to detect a species of fungus implicated in pulmonary fungal infections, our studies address a clinical problem using sound analytical chemistry. Ultimately, we hope these methods will be implemented into clinical labs for purposes of fungal diagnostics.

Supplementary Information The online version contains supplementary material available at https://doi.org/10.1007/s00216-021-03225-7.

Funding C.A. was supported by the National Institutes of Health $\mathrm{NIH}$ 5R01HL140301-02). A.M was supported by Biomedical Research Training for Veterinarians (NIH 9-T32 OD0-10437-18), NIH/NCATS Colorado CTSA (TL1 TR002533), and EveryCat Health Foundation (W20-032).

\section{Declarations}

Conflict of interest The authors declare no conflict of interest.

Ethics approval Not applicable; no human/animal studies were performed during the course of our experiments.

Source of biological material The source of cells used is covered in Section 2 Materials and methods under Materials.
Statement on animal welfare Hair collection from shelter-owned cats has been approved by Colorado State University's Clinical Review Board and has been granted an Institutional Animal Care and Use Committee (IACUC) waiver. The veterinarians at the collaborating shelters have given consent for hair sample collection from their shelter-owned cats and performed the collections themselves.

Open Access This article is licensed under a Creative Commons Attribution 4.0 International License, which permits use, sharing, adaptation, distribution and reproduction in any medium or format, as long as you give appropriate credit to the original author(s) and the source, provide a link to the Creative Commons licence, and indicate if changes were made. The images or other third party material in this article are included in the article's Creative Commons licence, unless indicated otherwise in a credit line to the material. If material is not included in the article's Creative Commons licence and your intended use is not permitted by statutory regulation or exceeds the permitted use, you will need to obtain permission directly from the copyright holder. To view a copy of this licence, visit http://creativecommons.org/licenses/by/4.0/.

\section{References}

1. Hesse SJA, Ruijter GJG, Dijkema C, Visser J. Intracellular $\mathrm{pH}$ homeostasis in the filamentous fungus Aspergillus niger. Eur J Biochem. 2002[cited 2020 Aug 23];269(14):3485-94. Available from: http://doi.wiley.com/10.1046/j.1432-1033.2002.03042.x.

2. Delmas S, Pullan ST, Gaddipati S, Kokolski M, Malla S, Blythe MJ, et al. Uncovering the genome-wide transcriptional responses of the filamentous fungus Aspergillus niger to lignocellulose using RNA sequencing. Nielsen J, editor. PLoS Genet. 2012 [cited 2020 Aug 23];8(8):e1002875. Available from: https://dx.plos.org/ 10.1371/journal.pgen.1002875.

3. Patterson JE, Peters J, Calhoon JH, Levine S, Anzueto A, AlAbdely $\mathrm{H}$, et al. Investigation and control of aspergillosis and other filamentous fungal infections in solid organ transplant recipients. Transpl Infect Dis. 2000 [cited 2020 May 17];2(1):22-8. Available from: http://doi.wiley.com/10.1034/j.1399-3062.2000.020105.x.

4. Vinck A, Terlou M, Pestman WR, Martens EP, Ram AF, Van Den Hondel CAMJJ, et al. Hyphal differentiation in the exploring mycelium of Aspergillus niger. Mol Microbiol. 2005;58(3):693-9.

5. Hocking AD. Aspergillus and related teleomorphs. In: Food spoilage microorganisms. Elsevier Ltd; 2006. p. 451-87.

6. Home - Aspergillus niger v1.0. [cited 2020 Aug 23]. Available from: https://mycocosm.jgi.doe.gov/Aspni1/Aspni1.home.html.

7. Gordon CL, Archer DB, Jeenes DJ, Doonan JH, Wells B, Trinci APJ, et al. A glucoamylase::GFP gene fusion to study protein secretion by individual hyphae of Aspergillus niger. J Microbiol Methods. 2000;42(1):39-48.

8. Ladaniya MS. Postharvest diseases and their management. In: Citrus Fruit. Elsevier; 2008. p. 417-XIX.

9. Person AK, Chudgar SM, Norton BL, Tong BC, Stout JE. Aspergillus niger: an unusual cause of invasive pulmonary aspergillosis. J Med Microbiol [Internet]. 2010 Jul [cited 2020 Aug 23];59(7):834-8. Available from: https://www.ncbi.nlm.nih. gov//pmc/articles/PMC3052473/?report=abstract.

10. Dagenais TRT, Keller NP. Pathogenesis of Aspergillus fumigatus in invasive aspergillosis [Internet]. Vol. 22, Clinical Microbiology Reviews. American Society for Microbiology (ASM); 2009 [cited 2020 Aug 23]. p. 447-65. Available from: https://www.ncbi.nlm. nih.gov//pmc/articles/PMC2708386/?report=abstract.

11. Paulussen C, Hallsworth JE, Álvarez-Pérez S, Nierman WC, Hamill PG, Blain D, et al. Ecology of aspergillosis: insights into the pathogenic potency of Aspergillus fumigatus and some other 
Aspergillus species. Vol. 10, Microbial Biotechnology. John Wiley and Sons Ltd; 2017 [cited 2020 Aug 23]. p. 296-322. Available from: https://www.ncbi.nlm.nih.gov//pmc/articles/PMC2708386/? report=abstract.

12. Hoshino H, Tagaki S, Kon H, Shibusa T, Takabatake H, Fujita A, et al. Allergic bronchopulmonary aspergillosis due to Aspergillus niger without bronchial asthma. Respiration. 1999 [cited 2020 Aug 23];66(4):369-72. Available from: https://www.karger.com/ Article/FullText/29392.

13. Center for Disease Control. Aspergillosis Statistics | Aspergillosis | Types of Fungal Diseases | Fungal Diseases | CDC. [cited 2020 Aug 23]. Available from: https://www.cdc.gov/fungal/diseases/ aspergillosis/statistics.html.

14. Ohno N. Comprehensive glycoscience. Volume 2: Analysis of Glycans, Polysaccharide Functional Properties. Elsevier Inc.; 2007.

15. Verma D, Fortunati E. Biopolymer processing and its composites: an introduction. In: Biomass, biopolymer-based materials, and bioenergy: Construction, Biomedical, and other Industrial Applications. Elsevier; 2019. p. 3-23.

16. Bhat GS, Rong H. Biodegradable nonwovens. In: Biodegradable and sustainable fibres: a volume in Woodhead Publishing Series in Textiles. Elsevier Ltd; 2005. p. 310-42.

17. Lopez-Romero E, Ruiz-Herrera J. The role of chitin in fungal growth and morphogenesis. In: Chitin in Nature and Technology. Springer US; 1986. p. 55-62.

18. Resh VH. Encyclopedia of Insects. Encyclopedia of insects. Elsevier Inc.; 2009.

19. Das S, Roy D, Sen R. Utilization of chitinaceous wastes for the production of chitinase. In: Advances in food and nutrition research. Academic Press Inc.; 2016. p. 27-46.

20. Lenardon MD, Munro CA, Gow NAR. Chitin synthesis and fungal pathogenesis. Vol. 13, Current Opinion in Microbiology. Elsevier; 2010. p. 416-23.

21. Winkler S, Kaplan DL. Biosynthesized materials: properties and processing. Encycl Mater Sci Technol 2001;609-15.

22. Pochanavanich P, Suntornsuk W. Fungal chitosan production and its characterization. Lett Appl Microbiol. 2002;35(1):17-21.

23. Veríssimo C. Fungal infections. In: Environmental mycology in public health: fungi and mycotoxins risk assessment and management. Elsevier Inc.; 2015. p. 27-34.

24. Pfaller MA, Diekema DJ. Rare and emerging opportunistic fungal pathogens: concern for resistance beyond Candida albicans and Aspergillus fumigatus. Vol. 42, Journal of Clinical Microbiology. American Society for Microbiology Journals; 2004. p. 4419-31.

25. GAFFI. Fungal Disease Frequency $\mid$ Gaffi - Global Action Fund for Fungal Infections. 2019 [cited 2020 Apr 12]. Available from: https://www.gaffi.org/why/fungal-disease-frequency/.

26. Badiee P, Hashemizadeh Z. Opportunistic invasive fungal infections: diagnosis \& clinical management. Vol. 139, Indian Journal of Medical Research. Indian Council of Medical Research; 2014. p. 195-204.

27. Rautemaa-Richardson R, Richardson MD. Systemic fungal infections. Vol. 45, Medicine (United Kingdom). Elsevier Ltd; 2017. p. 757-62.

28. Wenzel RP. Nosocomial candidemia: risk factors and attributable mortality. Clin Infect Dis. 1995;20(6):1531-4.

29. Denning DW. Invasive Aspergillosis. [cited 2020 Apr 12]. Available from: https://about.jstor.org/terms

30. Fungi and Fungal Infections | Gaffi - Global Action Fund for Fungal Infections. [cited 2020 Mar 9]. Available from: https://www.gaffi. org/why/fungi-fungal-infections/.

31. Bongomin F, Gago S, Oladele RO, Denning DW. Global and multi-national prevalence of fungal diseases - estimate precision. Vol. 3, J Fungi. 2017.
32. Bajaj SK, Tombach B. Respiratory infections in immunocompromised patients: lung findings using chest computed tomography. Radiol Infect Dis [Internet]. 2017;4(1):29-37. https://doi.org/10. 1016/j.jrid.2016.11.001.

33. Pragman AA, Berger JP, Williams BJ. Understanding persistent bacterial lung infections: clinical implications informed by the biology of the microbiota and biofilms. Clin Pulm Med. 2016;23(2): 57-66.

34. Pashley CH, Fairs A, Morley JP, Tailor S, Agbetile J, Bafadhel M, et al. Routine processing procedures for isolating filamentous fungi from respiratory sputum samples may underestimate fungal prevalence. Med Mycol. 2012 [cited 2020 Oct 5];50(4):433-8. Available from: https://academic.oup.com/mmy/article-lookup/doi/10.3109/ 13693786.2011 .615762$.

35. van Woerden HC, Gregory C, Brown R, Marchesi JR, Hoogendoorn B, Matthews IP. Differences in fungi present in induced sputum samples from asthma patients and non-atopic controls: a community based case control study. BMC Infect Dis. 2013 [cited 2020 Oct 5];13(1):1-6. Available from: https://link.springer. com/articles/10.1186/1471-2334-13-69

36. Pan H. A non-covalent dimer formed in electrospray ionisation mass spectrometry behaving as a precursor for fragmentations. Rapid Commun Mass Spectrom. 2008 [cited 2020 Apr 14];22(22):3555-60. Available from: http://www.ncbi.nlm. nih.gov/pubmed/18853406.

37. Schug K, McNair HM. Adduct formation in electrospray ionization mass spectrometry: II. Benzoic acid derivatives. J Chromatogr A. 2003;985(1-2):531-9.

38. Smith RD, Light-Wahl KJ, Winger BE, Loo JA. Preservation of non-covalent associations in electrospray ionization mass spectrometry: multiply charged polypeptide and protein dimers. Org Mass Spectrom. 1992 [cited 2020 Apr 14];27(7):811-21. Available from: http://doi.wiley.com/10.1002/oms.1210270709.

39. Li J, Revol J, Marchessault RH. Effect of degree of deacetylation of chitin on the properties of chitin crystallites. 1996;373-80.

40. Mbareche H, Veillette M, Teertstr W, Kegel W, Bilodeau GJ, Wösten HAB, et al. Recovery of fungal cells from air samples: a tale of loss and gain. Appl Environ Microbiol [Internet]. 2019 May 1 [cited 2020 Oct 9];85(9). Available from: https://www. ncbi.nlm.nih.gov//pmc/articles/PMC6495771/?report=abstract.

41. Song M, Hang TJ, Wang C, Yang L, Wen AD. Precolumn derivatization LCMS/MS method for the determination and pharmacokinetic study of glucosamine in human plasma and urine. J Pharm Anal. 2012 [cited 2021 Jan 9];2(1):19-28. Available from: https:// www.ncbi.nlm.nih.gov//pmc/articles/PMC5760825/?report= abstract.

42. Liu Y, Li Z, Liu G, Jia J, Li S, Yu C. Liquid chromatographytandem mass spectrometry method for determination of $\mathrm{N}$ acetylglucosamine concentration in human plasma. J Chromatogr B Anal Technol Biomed Life Sci. 2008 [cited 2021 Jan 9];862(12):150-4. Available from: https://pubmed.ncbi.nlm.nih.gov/ 18165162

43. Roda A, Sabatini L, Barbieri A, Guardigli M, Locatelli M, Violante FS, et al. Development and validation of a sensitive HPLC-ESIMS/MS method for the direct determination of glucosamine in human plasma. J Chromatogr B Anal Technol Biomed Life Sci. 2006 Nov 21;844(1):119-26.

Publisher's note Springer Nature remains neutral with regard to jurisdictional claims in published maps and institutional affiliations. 